\title{
Zinc transporter SLC39A7/ZIP7 is essential for intestinal homeostatic self- renewal
}

Wakana Ohashi ${ }^{1}$, Koji Hase ${ }^{2}$, Toshiyuki Fukada ${ }^{3}$

${ }^{I}$ Department of Molecular and Medical Pharmacology, Graduate School and Pharmaceutical Sciences, University of Toyama, Japan, ${ }^{2}$ Division of Biochemistry, Faculty of Pharmacy, Keio University, Japan, ${ }^{3}$ Molecular and Cellular Physiology, Faculty of Pharmaceutical Sciences, Tokushima Bunri University, Japan

The essential trace element zinc is required for the normal functions of the many organs including the intestine. Dysregulation of zinc homeostasis has been implicated in disorders such as intestinal inflammation and aberrant epithelial morphology. However, the molecular mechanisms by which zinc regulates intestinal physiology are largely unknown. A zinc-uptake transporter, Zrt-Irt-like protein 7 (ZIP7) is highly expressed in the intestinal crypt, but little is known about its role. Hence, we aimed to investigate the role of ZIP7 in intestinal epithelium in mice.

We assessed the expressions of ZIP7 in intestinal epithelium by qPCR, western blot, and in situ hybridization analysis. We generated the mice with an inducible, intestinal epithelium-specific knockout of Zip7. To investigate the role of Zip7 in crypt cells, Intestinal stem cells and transit-amplifying (TA) cells from Zip7-deficient mice were isolated and subjected to gene expression analysis.

In situ hybridization analysis revealed that Zip7 was distributed in the middle and lower crypt regions in a pattern much like that of TA cells. Immunohistochemistry confirmed that Zip7-expressing cells were positive for Ki67, a marker for TA cells. Furthermore, Zip7 was also expressed in Lgr5+ stem cells, and post-mitotic Paneth cells. Zip7 deletion in intestinal epithelium caused a significant impairment of epithelial integrity. Electron microscopy revealed that Zip7 deletion triggered the death of massive numbers of TA cells. This abnormality was accompanied with loss of intestinal stem cells, suggesting an essential role of Zip7 in TA cell proliferation and stem cell maintenance. In gene expression profiling, unfolded protein response (UPR)-related genes were highly upregulated in the Zip7-deficient TA cells.

UPR plays a key role in regulation of proliferative property of intestinal epithelium, whereas excessive UPR evokes unresolved ER stress. Our data demonstrated that Zip7-deficiency abnormally increases ER stress, leading to the massive death of TA cells followed by the loss of intestinal stem cells. These observations uncover a novel link between intracellular zinc regulation by ZIP7 and resolving ER stress to maintain homeostatic self-renewal of intestinal epithelium. 